\title{
Valor predictivo positivo de BI-RADS e indicaciones en resonancia magnética de mama, experiencia de un centro de alta complejidad
}

\author{
Positive predictive value of BI-RADS and indications in breast MRI, the experience of a highly complex center
}

Autores:

González E Martha'; Dávila V Claudia²; Juan S Isaac²; Vieira S Santiago³; Estrada O Kelly
${ }^{1}$ Fundación Universitaria Sanitas, Departamento de Radiología e Imágenes diagnósticas. Bogotá, Colombia.
${ }^{2}$ Fundación Universitaria Sanitas, Postgrado de Radiología e Imágenes diagnósticas. Bogotá, Colombia.
${ }^{3}$ Fundación Universitaria Sanitas, Departamento de Ginecología y Obstetricia. Bogotá, Colombia.
${ }^{4}$ Fundación Universitaria Sanitas, Instituto de Investigaciones Clínicas, Facultad de Medicina. Bogotá, Colom-
bia.

Resumen: La mamografía y el ultrasonido constituyen el pilar para el estudio del cáncer de mama. Otras modalidades como la resonancia magnética (RM) de mama no tiene un alto nivel de evidencia para sus indicaciones. El objetivo de este estudio es determinar las indicaciones de solicitud de RM de mama y el valor predictivo positivo (VPP) de la resonancia frente al diagnóstico histológico y lesiones modificadoras de la conducta clínica. Se realizó un estudio analítico, de corte transversal que incluyó pacientes femeninas valoradas con RM de mama entre mayo 2017-noviembre 2018 en un centro especializado de patología de mama en la Clínica Universitaria Colombia en Bogotá. Se calcularon los VPP de las indicaciones de RM de mama modificadores de la conducta clínica (BI-RADS 4,5,6) y los VPP de las mismas frente a hallazgos histológicos.

Se incluyeron 236 mujeres con RM de mama, la prevalencia de cáncer de mama fue 49.57\%, entre las indicaciones que se reconocieron "evaluación de la extensión de la enfermedad" $19.49 \%$ y otras indicaciones no soportadas en la literatura 35.59\%, fueron las más frecuentes. El VPP para cáncer en BI-RADS 4 fue $29 \%$, BI-RADS 5 79\% y para BI-RADS 4 y 5 de 60\%. EI VPP para cáncer en pacientes de alto riesgo fue de $12.82 \%$. Las indicaciones para solicitud de RM basadas y no basadas en la evidencia tienen un bajo VPP aun en un escenario de alta prevalencia de cáncer de mama. Se requiere una mejor caracterización del riesgo y descripción de las indicaciones para solicitud de RM.

Abstract: Mammography and ultrasound are the mainstay for the study of breast cancer. Other modalities such as magnetic resonance imaging (MRI) of the breast do not have a high level of evidence for their indications. The objective of this study is to determine the indications for requesting breast MRI and the positive predictive value (PPV) of the resonance against histological diagnosis and lesions modifying clinical behavior. An Analytical, cross-sectional study that included female patients assessed with breast MRI between May 2017-November 2018 in a specialized center for breast pathology in Colombia in the ClínicaUniversitaria Colombia in Bogotá, Colombia. The PPV of the indications of clinical conduct modifying breast MRI $(B I-R A D S 4,5,6)$ and the PPV of the same were calculated against histological findings.

236 women with breast MRI were included, the prevalence of breast cancer was 49.57, among the indications that were recognized as "evaluation of the extension of the disease" $19.49 \%$ and other indications not supported in the literature (other reason) $35.59 \%$, were the most frequent. The PPV for cancer in BI-RADS 4 was $29 \%$, BI-RADS 5 79\% and for BI-RADS 4 and 5 60\%. The PPV for cancer in high-risk patients was $12.82 \%$.

The evidence-based and non-evidence-based indications for MRI requests have a low PPV even in a scenario of high prevalence of breast cancer. A better characterization of the risk and description of the indications for $M R$ request are required.
Palabras clave (DeCs): Imagen por resonancia magnética, neoplasias de la mama, diagnostico, cribado.

Key words (MeSH): Magnetic resonance imaging, breast neoplasms, diagnostic, screening.

Comité de ética: Este estudio fue comunicado y aprobado por el Departamento de Radiología e Imágenes diagnósticas. Fundación Universitaria Sanitas, Bogotá, Colombia.

\section{Correo para correspondencia} del autor principal:

Dr. Isaac Alfonso Juan Sierra, isjuan91@hotmail.com, ijuanmd@ hotmail.com

\section{Fecha de recepción:}

13 de febrero de 2020

Fecha de aceptación:

09 de septiembre de 2020

\section{Introducción}

El cáncer de mama es un problema de salud pública a nivel mundial con 2.1 millones de casos nuevos anuales y es la principal causa de muerte por cáncer en mujeres. La mortalidad para el año 2018 fue de 626.679 representando un $29.8 \%$ de todas las muertes por cáncer de mama en mujeres. La incidencia es variable oscilando entre 27-92 casos por 100.000 mujeres, siendo mayor en los países con alto índice de desarrollo humano (IDH).

En Latinoamérica y el caribe el cáncer de mama ocupa el primer puesto en cáncer teniendo en cuenta todas las edades y ambos sexos con 51.9 casos por 100.000 habitantes, con una prevalencia de 156.743 y una mortalidad de 52.558 casos en 2018. En Colombia, la incidencia es de 44 casos por cada 100.000 habitantes, con una prevalencia de 10.023 casos nuevos y una mortalidad de 3702 casos en $2018 .^{1,3}$

El cáncer de mama se estudia mediante la mamografía, la ecografía y el análisis histológico mediante biopsias guiadas por distintos métodos ${ }^{4}$. El estudio de tumores de mama por resonancia magnética se comenzó a introducir en el año 1982 y desde el año 2003, se posiciona como una modalidad diagnóstica hecho que concuerda, con la publicación de la cuarta edición del BI-RADS ${ }^{5}$. El aumento en la frecuencia de su uso coincide con la aparición de las primeras recomendaciones por parte del Colegio Americano de Radiología. La resonancia magnética es reconocida en general por la capacidad de contraste en los tejidos blandos que posee, característica de suma importancia en la mama dada su composición grasa $y$ de tejido glandular. ${ }^{6}$ 
El uso de medio de contraste, en conjunto a la supresión grasa y la sustracción de imágenes, aumenta la sensibilidad en la detección de cáncer, debido a la neovascularización que este tipo de lesiones suelen tener. ${ }^{7}$ Adicional al realce de las lesiones, se ha descrito que la curva de lavado de las lesiones se relaciona con su potencial maligno, teniendo mayor frecuencia de malignidad aquellas lesiones que presentan realce temprano y de predominio periférico, con curvas de lavado rápidas, permitiendo diferenciar algunas lesiones benignas de las malignas. ${ }^{8}$

Las secuencias de difusión y ADC se basan en el movimiento aleatorio de las moléculas de agua, dicho movimiento disminuye cuando aumenta el número de células en un determinado volumen de tejido lo cual es característico de los tumores malignos. Esto se manifiesta como hiperintensidad en la secuencia de DWI y disminución de los valores en el mapa de ADC.

Un metaanálisis basado en 13.847 lesiones concluye que un valor menor de $1.00 \times 10-3 \mathrm{~mm} 2 / \mathrm{s}$ puede ser recomendado para distinguir lesiones malignas de benignas. $^{9}$

A pesar de contar con muchas características positivas, principalmente cuando su uso está indicado, la resonancia magnética de mama tiene algunos inconvenientes en su aplicación como los altos costos, en promedio de 2,925 USD en Estados Unidos ${ }^{10}$, uso de gadolinio como medio de contraste, el cual puede producir efectos adversos como reacciones alérgicas, depósito en el cerebro, generar fibrosis nefrogénica sistémica, entre otros ${ }^{11,13}$, y en términos de pronóstico, no se ha podido demostrar en los estudios que su uso se asocie a disminución en la mortalidad por cáncer de mama. ${ }^{14}$

El uso indiscriminado de esta modalidad en los últimos años, ha generado que grupos de expertos desincentiven su uso masivo y se generen recomendaciones para el adecuado uso de esta modalidad de imagen. ${ }^{15-19}$

A pesar de estos esfuerzos, existe gran variabilidad en la práctica clínica, con indicaciones de uso que no se encuentran soportadas en evidencia, como el estadiaje prequirúrgico del cáncer de mama de reciente diagnóstico, la evaluación de los efectos de la quimioterapia neoadyuvante, resolución de incertidumbre cuando hay hallazgos contradictorios o poco específicos entre la mamografía y el ultrasonido, ante la sospecha de invasión a la fascia por lesiones, sospecha de recurrencia local de lesión cuando la biopsia no pueda ser realizada, entre otras. ${ }^{20}$
En la actualidad, la indicación principal, es el cribado en pacientes de alto riesgo, definido como:

1. Mujeres con mutación genética conocida a quienes se les debe ofrecer vigilancia anual si son: portadoras de mutaciones BRCA 1 y BRCA 2 con edad entre 30 a 49 años y/o portadoras de mutación TP53 mayores de 20 años.

2. Mujeres de 30 a 49 años con riesgo a 10 años igual o mayor al $20 \%$ o a mujeres de 40 a 49 años con riesgo a 10 años igual o mayor al $12 \%$ cuando la mamografía ha mostrado un patrón de mamas densas.

3. Mujeres entre los 30 a 49 años quienes no han sido evaluadas genéticamente, pero quienes tienen un alto chance de ser portadoras de mutaciones genéticas BRCA1 o TP53 si ellas están en: un riesgo del $50 \%$ de portar alguna de estas mutaciones en una familia evaluada o un riesgo de portar estas mutaciones del $50 \%$ en una familia no eva-luada ó inconclusamente evaluada con al menos un chance del $60 \%$ de portar la mutación para los genes BRCA 1 y TP $53^{20}$. Otras indicaciones no tienen evidencia de cambiar el curso clínico. ${ }^{20,21}$

\section{Objetivo}

Dado la heterogeneidad en las indicaciones que se encuentran en la práctica cotidiana, y los vacíos de conocimiento sobre el rendimiento de la RM de mama, se realiza este estudio con el objetivo de demostrar si las indicaciones descritas en la literatura justifican su uso, a partir del cálculo de los valores predictivos de las mismas frente a cambios de conducta clínica por hallazgos (BIRADS 4-5) o frente a hallazgos histológicos.

\section{Métodos}

Se trata de un estudio analítico de corte transversal, basado en datos retrospectivos, para determinar el rendimiento de la resonancia magnética de mama, medido a partir de su valor predictivo positivo en cada una de las indicaciones basadas y no basadas en evidencia de acuerdo con la clasificación de González y cols. ${ }^{20}$

El estudio fue llevado a cabo en una institución de alta complejidad, en la unidad de mama que tiene como función el estudio integral, diagnóstico y manejo de las lesiones de mama, en hombres y mujeres en Bogotá Colombia. 
La unidad está compuesta por múltiples especialidades médicas y quirúrgicas: Ginecología y obstetricia, radiología e imágenes diagnósticas, cirugía de seno y de tejidos blandos, cirugía plástica y recons tructiva, oncología clínica.

Para el estudio se seleccionaron mediante muestreo consecutivo estricto los reportes, historia clínica e imágenes de pacientes femeninas entre 18 años o más, que fueron atendidas en la unidad de mama de la Clínica entre el 01 de mayo del 2017 hasta el 01 de noviembre del 2018. Solo se excluyeron las pacientes cuya solicitud de resonancia magnética de mama tuviera como objetivo la valoración y seguimiento de prótesis mamaria o se tuvieran datos incompletos.

Se recolectó información para el estudio a partir de tres fuentes:

I. Las historias clínicas en el sistema electrónico SO$\mathrm{FIA}^{\circledR}$, de las cuales se obtuvieron las indicaciones que soportaban la solicitud de la imagen diagnóstica,

II. El sistema electrónico de imágenes IMPAX ${ }^{\circledR}$, donde se obtuvieron las imágenes de resonancia y sus respectivos informes que contenían información como motivo de solicitud, edad, factores de riesgo, técnica, hallazgos conclusión y clasificación BI-RADS; y

III. El sistema electrónico ARES ${ }^{\circledR}$, del que se obtuvo la información relacionada con reportes de patología, en los casos en los que se verificó la clasificación histológica de las lesiones, cuando se indicó biopsia.

Los estudios de resonancia magnética de mama fueron obtenidos en un equipo General Electric (GE) de 1.5T, la bovina utilizada fue una (GE) HDxBreast de ocho canales. Estos estudios incluyeron secuencias ponderadas en T1 y T2, así como secuencias con saturación grasa, difusión y sagital dinámico de 8 fases VIBRANT posterior a la administración de Gadobutrol como medio de contraste para magnético a un flujo de $3 \mathrm{cc} / \mathrm{seg}$ con un volumen de $0.2 \mathrm{mmol} / \mathrm{kg}$ de peso.

Los datos de la historia clínica y las imágenes fueron revisados por dos radiólogos con más de 10 años de experiencia en el diagnóstico de lesiones mamarias, utilizando el software de lectura asistida CADS$\operatorname{TREAM}^{\circledR}$ de General Electric.

Los datos fueron recolectados en una hoja de cálculo estandarizada en donde todos los investigadores recolectaron los datos con las mismas variables con el fin de garantizar la homogeneidad de la muestra.
Se extrajo información relacionada con la edad, las indicaciones, el informe de lectura de resonancia por radiología, el BI-RADS asignado y el resultado de patología.

Se realizó la estadística descriptiva de los sujetos participantes de la población de estudio, las variables cualitativas son presentadas como medidas de frecuencia relativa o frecuencia absoluta y las variables de tipo cuantitativo son presentadas como medidas de tendencia central y dispersión de acuerdo con la naturaleza y distribución de las variables.

Se calculó el valor predictivo positivo a partir de los verdaderos positivos más falsos positivos de las siguientes indicaciones: tamización de pacientes con alto riesgo, resolución de problemas cuando los hallazgos no concuerdan entre la mamografía y el ultrasonido, y bajo la indicación de "otro motivo" en las cuales se les solicitó resonancia por algún otro motivo no descrito o soportado en la literatura actual.

Todos los resultados son presentados con intervalos de confianza del $95 \%$ y el análisis estadístico se realizó con el software STATA $14^{\circledR}$.

El estudio fue aprobado por el comité de ética me diante el acta CEIFUS 386-18 el 26 de junio del 2018 de la Fundación Universitaria Sanitas.

\section{Resultados}

En total se obtuvieron 253 pacientes, de los cuales se excluyeron 17 pacientes por datos incompletos en las historias clínicas o por no contar con las imágenes en el software de radiología, obteniendo un total de 236 pacientes (Figura 1). El promedio de edad fue de 55 años siendo la menor de 22 años y la mayor de 86 años. La prevalencia de cáncer de mama en la muestra analizada fue de $49.57 \%$ (IC95= 43.2-56\%).

Delas236 pacientes, enel $16.53 \%(n=39$, IC95\% $=11.8$ $21.3 \%$ ) la resonancia magnética de mama fue indicada para la tamización de pacientes con alto riesgo, en el $18.64 \%(n=44, I C 95 \%=13.7-23.6 \%)$ para estudio de estadiaje de cáncer de reciente diagnóstico, en el $19.49 \%(n=46, I C 95 \%=14.4-24.5 \%)$ se solicitó para evaluación de la extensión de una lesión maligna, en el $5.53 \%$ de las pacientes $(n=13$, IC95\% $=2.6-8.4 \%)$ se solicitó para dirimir o confirmar resultados incongruentes entre la mamografía y el ultrasonido y en el $0.42 \%(n=1$, IC $95 \%=0-1.3 \%)$ estuvo indicado por sospecha de recaída local cuando la biopsia no pudo ser realizada. 
Figura. 1: Algoritmo de selección y características radiológicas de muestra analizada

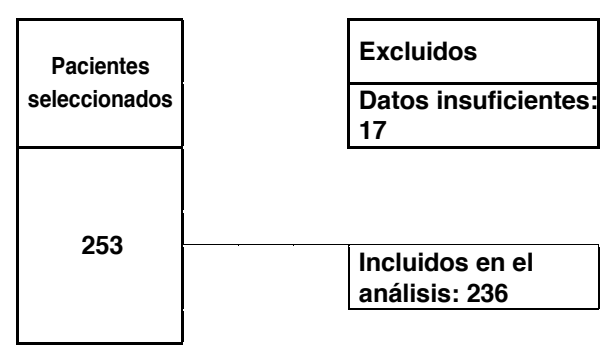

\begin{tabular}{|c|c|c|c|c|c|c|c|}
\hline & $\begin{array}{c}\text { BIRADS } \\
0 \\
\end{array}$ & $\begin{array}{c}\text { BIRADS } \\
1 \\
\end{array}$ & $\begin{array}{c}\text { BIRADS } \\
2 \\
\end{array}$ & $\begin{array}{c}\text { BIRADS } \\
3 \\
\end{array}$ & $\begin{array}{c}\text { BIRADS } \\
4 \\
\end{array}$ & $\begin{array}{c}\text { BIRADS } \\
5 \\
\end{array}$ & $\begin{array}{c}\text { BIRADS } \\
6 \\
\end{array}$ \\
\hline $\begin{array}{c}\text { Tamizaje de pacientes con alto } \\
\text { riesgo: } 39\end{array}$ & 0 & 3 & 20 & 11 & 3 & 1 & 1 \\
\hline $\begin{array}{l}\text { Estadiaje de reciente } \\
\text { diagnóstico: } 44\end{array}$ & 0 & 0 & 0 & 0 & 1 & 6 & 37 \\
\hline $\begin{array}{c}\text { Evaluación de la terapia } \\
\text { neoadyuvante: } 9\end{array}$ & 0 & 0 & 0 & 0 & 0 & 0 & 9 \\
\hline Recaída local sin biopsia: 1 & 0 & 0 & 0 & 0 & 0 & 1 & 0 \\
\hline $\begin{array}{c}\text { resultados incongruentes } \\
\text { entre ecografía y mamografía: } \\
13\end{array}$ & 0 & 3 & 4 & 2 & 3 & 1 & 0 \\
\hline Evaluación de la extensión: 46 & 1 & 0 & 2 & 0 & 1 & 5 & 37 \\
\hline $\begin{array}{l}\text { Carcinoma oculto, invasión a } \\
\text { la fascia y bordes positivos } \\
\text { post cuadrantectomia: } 0\end{array}$ & 0 & 0 & 0 & 0 & 0 & 0 & 0 \\
\hline Otras indicaciones: 84 & 1 & 7 & 40 & 9 & 9 & 10 & 8 \\
\hline
\end{tabular}

Fuente: Datos propios de los autores

$(\mathrm{N}=44)$ y el "estadiaje de reciente diagnostico" $(\mathrm{N}=46)$ obtuvo el mayor número de pacientes reportados con la categoría de BI-RADS 6, representando el $84 \%$ y $80.43 \%(n=37$ IC95\%= 73.3-94.9\%) $(n=37, I C 95 \%=$ $69-91.9 \%)$ respectivamente, sin embargo, se obtuvo 1 reporte clasificado como BI-RADS 4 (2.17\%) (13.04\%) y 6 pacientes reportados como BI-RADS 5.

Para la evaluación de la extensión, se obtuvo de forma similar un paciente clasificado como BIRADS 4 y 5 pacientes reportados como BI-RADS 5 lo que indica que el $16 \%$ ( 7 pacientes) de los pacientes en quienes se les solicitó la resonancia magnética de mama para estadiaje de reciente diagnóstico y el 13\% (6 pacientes) de los pacientes en quienes se les solicitó para evaluar la extensión de la enfermedad no contaban con un diagnóstico histopatológico al momento de realización del estudio, aunque así lo sugiriera la indicación.

La mayor cantidad de pacientes reportados como BIRADS 2 fueron aquellas mujeres a quienes se les solicito el estudio por otro "motivo" ( $\mathrm{N}=84)$ representando un $47.61 \%$ ( $n=40$ IC95\% 36.9-58.3\%).

En la tabla 3 se representan las diferentes categorías BI-RADS asignadas por motivos de solicitud incluyendo "otro motivo"(cualquier otro motivo diferente a las recomendaciones vigentes).

El motivo de solicitud de "evaluación de la extensión" 
De los 17 pacientes con resonancia reportada como BI-RADS 4, 5 fueron positivas para cáncer obteniendo un Valor predictivo positivo (VPP) de $29 \%$ (IC95\%=7.8-51.1\%). De los 24 pacientes con resonancia reportada como BI-RADS 5, 19 fueron positivas para cáncer obteniendo un VPP de 79\% (IC95\% $=62.9-95.4 \%$ ) y cuando se combinan ambas categorías se obtuvo un VPP de 60\% (IC95\%=46-75.9\%).

La tabla 4 ilustra los valores predictivos para cáncer de mama en resonancias clasificadas como BI-RADS $4 \circ 5$.

El VPP de la resonancia magnética de las indicaciones basadas en la evidencia (tamización de pacientes con alto riesgo) fue heterogéneo, encontrando que para detección de lesiones clasificadas como BIRADS 4 fue de 7,69\%, para lesiones BIRADS 5 fue de 2,56\% y para lesiones BIRADS 6 fue de 2,56\%.

Esta indicación predijo tan solo el $12.82 \%$ de las lesiones que ameritan una intervención clínica, cuando se estudia con resonancia.

Para las indicaciones sin evidencia, en dónde la resonancia magnética podía impactar en un cambio en la conducta como la resolución de problemas cuando los hallazgos no concuerdan entre la mamografía y el ultrasonido, se encontró que el VPP para encontrar una lesión BI-RADS 4 fue de $23.07 \%$ y para lesiones BI-RADS 5 fue de $7.69 \%$.

Esta indicación predijo $30.76 \%$ de las lesiones que ameritan una intervención clínica, cuando se estudia con resonancia magnética.

En la tabla 6 se detallan los VPP de otras indicaciones para encontrar lesiones BI-RADS 4 y 5.

\section{Discusión}

Este trabajo tuvo como objetivo demostrar si las indicaciones soportadas y no soportadas en la evidencia justifican el uso de resonancia magnética a partir del cálculo de los valores predictivos, para cada una de las indicaciones.

Los valores predictivos positivos para cáncer de mama de la RM para lesiones catalogadas como BIRADS 4 y 5 fueron de 29\% (IC95=7.8\%-51.1\%) para el BI-RADS 4 y de $79 \%$ (IC95=62.9\%-95.4\%) para BIRADS 5.

Estos resultados son similares a los que reportan otros autores que encuentran un VPP para BI-RADS
4 de $20.5 \%$ (IC95=12.4-30.8\%) y para el BI-RADS 5 un VPP de $71.4 \%$ (IC95=41.9-91.6\%). ${ }^{22}$

También se encontró que cuando se realiza resonancia magnética bajo las indicaciones que pueden impactar la conducta clínica (tamización de pacientes con alto riesgo, resultados incongruentes entre la mamografía y el ultrasonido o cuando es solicitada por cualquier otro motivo clínico), los valores predictivos son variables: para la indicación de "tamización de pacientes con alto riesgo" el VPP para lesiones BIRADS 4 fue $7.69 \%$ (IC95=0-16.1\%), para el BI-RADS 5 de $2.56 \%$ (IC95=0-7.5\%) y el VPP para el cambio de conducta terapéutica cuando se utiliza la resonancia para esta indicación es de 12.82\% (IC95=2.3-23.3\%).

En este estudio cuando se utilizó la resonancia con indicación de resultados incongruentes entre la mamografía y el ultrasonido, el VPP para lesiones BIRADS 4 fue de $23.07 \%$ (IC95=0-46\%), para el BIRADS 5 fue de $76.9 \%$ (IC95=6.8-22.2\%) y el VPP para modificar la conducta bajo esta indicación fue de $30.76 \%$ (IC95=5.7-0.55.9\%).

Cuando se realizó la resonancia bajo la indicación de "otro motivo" el VPP para BI-RADS 4 fue de $10.71 \%$ (IC95 $=4.1-17.3 \%)$ para el BI-RADS 5 fue de $12.04 \%$ (IC95=5-18.8\%) y el VPP de cambio de la conducta fue de $32.53 \%$ (IC95=22.2-42.1\%).

De las indicaciones que cambian la conducta (alto riesgo y hallazgos incongruentes entre la mamografía y el ultrasonido y otros motivos) superaron por poco el VPP de $30 \%$ a pesar de que el estudio se realizó en la clínica universitaria Colombia que es una institución con alta prevalencia de cáncer de mama (49.57\%) lo cual puede interpretarse como un pobre rendimiento predictivo de la resonancia en estas indicaciones en nuestro medio.

Otro hallazgo que llama la atención es el porcentaje de pacientes a quienes se les pide el estudio bajo otras indicaciones (35.59\%), en donde el estudio se solicita por muchas otras razones diferentes a las que están descritas en la literatura, que tienen soporte basado en la evidencia, similar a lo que ocurre en Estados Unidos. ${ }^{18,19}$

En este estudio se encontraron algunas particularidades, la verdadera utilidad en términos de hacer un nuevo diagnostico o aumentar la sospecha es solo el $17 \%$ de los casos. 
Tabla 1. Indicaciones de solicitud de resonancia magnética:

\begin{tabular}{lcc}
\hline Variable & $\mathbf{n ~ ( \% )}$ & IC95 (\%) \\
\hline Tamizaje para pacientes de alto riesgo & $39(16.53 \%)$ & $11.8-21.3 \%$ \\
Estadiaje de reciente diagnóstico & $44(18.64 \%)$ & $13.7-23.6 \%$ \\
Evaluación del efecto neoadyuvante & $9(3.8139 \%)$ & $1.4-3.6 .3 \%$ \\
Carcinoma primario oculto & 0 & $\mathrm{~N} / \mathrm{A}$ \\
Sospecha de recaída local cuando la & $1(0.42 \%)$ & $0-1.3 \%$ \\
biopsia no pueda ser realizada & & \\
Resultados incongruentes* & $13(5.53 \%)$ & $2.6-8.4 \%$ \\
Evaluación de la extensión & $46(19.49 \%)$ & $14.4-24.5 \%$ \\
Invasión a la fascia & 0 & $\mathrm{~N} / \mathrm{A}$ \\
Bordes positivos & 0 & $\mathrm{~N} / \mathrm{A}$ \\
otro motivo** & $84(35.59 \%)$ & $29.5-41.7 \%$ \\
\end{tabular}

$\mathrm{N}=236$.

*Resultados incongruentes entre la mamografía y el ultrasonido.

** Otro motivo diferente a las indicaciones y/o recomendaciones actuales planteadas en la literaturapj "bajo riesgo para cáncer de mama, estudio por lesión dolorosa"

N/A: No aplica.

Fuente: Datos propios de los autores.

Tabla 2. Diagnóstico por RM de mama de acuerdo con el BI-RADS.

\begin{tabular}{lll}
$\mathbf{R M}$ & $\mathbf{n}(\%)$ & IC95 (\%) \\
\hline BI-RADS 0 & $2(0.85 \%)$ & $0.3-0.20 \%$ \\
BI-RADS 1 & $13(5.51 \%)$ & $2.6-8.4 \%$ \\
BI-RADS 2 & $66(27.96 \%)$ & $22.2-33.7 \%$ \\
BI-RADS 3 & $22(9.32 \%)$ & $5.6-13 \%$ \\
BI-RADS 4 & $17(7.2 \%)$ & $3.9-10.5 \%$ \\
BI-RADS 5 & $24(10.16 \%)$ & $6.3-14 \%$ \\
BI-RADS 6 & $92(38.98 \%)$ & $32.8-45.2 \%$ \\
\hline
\end{tabular}

$\mathrm{N}=236$.

Fuente: Datos propios de los autores. 
Tabla 3. Diagnóstico BIRADS por motivo de solicitud

\begin{tabular}{|c|c|c|c|c|c|c|c|c|}
\hline & N236, n(\%) (IC) & $\begin{array}{c}\text { BIRADS } \\
0\end{array}$ & $\begin{array}{l}\%(\mathrm{IC} \\
95 \%)\end{array}$ & $\begin{array}{c}\text { BIRADS } \\
1\end{array}$ & $\begin{array}{l}\% \text { (IC } \\
95 \%)\end{array}$ & \multicolumn{2}{|c|}{$\%$ (IC 95\%) } & \\
\hline $\begin{array}{l}\text { Tamizaje de } \\
\text { pacientes con alto } \\
\text { riesgo }\end{array}$ & $\begin{array}{l}39(16.53 \%) \\
(11.8-21.3 \%)\end{array}$ & 0 & $\mathrm{~N} / \mathrm{A}$ & 3 & $\begin{array}{c}7.69 \\
(0- \\
16.1 \%)\end{array}$ & 51,2 & $\begin{array}{c}51,28(35,6 \% \\
67 \%)\end{array}$ & \\
\hline $\begin{array}{l}\text { Estadiaje de } \\
\text { reciente } \\
\text { diagnóstico }\end{array}$ & $\begin{array}{l}44(18.64 \%) \\
(13.7-23.6 \%)\end{array}$ & 0 & $\mathrm{~N} / \mathrm{A}$ & 0 & $\mathrm{~N} / \mathrm{A}$ & 0 & $\mathrm{~N} / \mathrm{A}$ & \\
\hline $\begin{array}{l}\text { Evaluación de la } \\
\text { terapia } \\
\text { neoadyuvante }\end{array}$ & $\begin{array}{l}9(3.8139 \%) \\
(1.4-3.6 .3 \%)\end{array}$ & 0 & $\mathrm{~N} / \mathrm{A}$ & 0 & $\mathrm{~N} / \mathrm{A}$ & 0 & $\mathrm{~N} / \mathrm{A}$ & \\
\hline $\begin{array}{l}\text { Carcinoma } \\
\text { primariooculto }\end{array}$ & $0(0 \%)$ & 0 & $\mathrm{~N} / \mathrm{A}$ & 0 & $\mathrm{~N} / \mathrm{A}$ & 0 & $\mathrm{~N} / \mathrm{A}$ & \\
\hline $\begin{array}{l}\text { Sospecha de } \\
\text { recurrencia local } \\
\text { cuando la biopsia } \\
\text { no pueda ser } \\
\text { realizada }\end{array}$ & $\begin{array}{c}1(0.42 \%)(\theta \\
1.3 \%)\end{array}$ & 0 & $\mathrm{~N} / \mathrm{A}$ & 0 & $\mathrm{~N} / \mathrm{A}$ & 0 & N/A & \\
\hline $\begin{array}{l}\text { Resultados } \\
\text { incongruentes } \\
\text { entre la } \\
\text { mamografía y el } \\
\text { ultrasonido }\end{array}$ & $\begin{array}{c}13(5.53 \%)(2.6 \\
8.4 \%)\end{array}$ & 0 & $\mathrm{~N} / \mathrm{A}$ & 3 & $\begin{array}{l}23(0- \\
46 \%)\end{array}$ & $\begin{array}{r}30 \\
5\end{array}$ & $\begin{array}{c}30,56(5.7 \\
55.9 \%)\end{array}$ & \\
\hline $\begin{array}{l}\text { Evaluación de } \\
\text { extensión }\end{array}$ & $\begin{array}{r}46(19.49 \%) \\
(14.4-24.5 \%)\end{array}$ & 1 & $\begin{array}{c}2.17 \\
(0- \\
6.4 \%)\end{array}$ & 0 & $\mathrm{~N} / \mathrm{A}$ & $\begin{array}{l}4 \\
1\end{array}$ & $\begin{array}{l}4,34(0- \\
10.2 \%)\end{array}$ & \\
\hline $\begin{array}{l}\text { Invasión a la fascia } \\
\text { profunda. }\end{array}$ & $0 \% \theta$ & 0 & $\mathrm{~N} / \mathrm{A}$ & 0 & $\mathrm{~N} / \mathrm{A}$ & 0 & $\mathrm{~N} / \mathrm{A}$ & \\
\hline $\begin{array}{l}\text { Bordes positivos } \\
\text { posterior a la } \\
\text { cuadrantectomía. }\end{array}$ & $0 \% \theta$ & 0 & $\mathrm{~N} / \mathrm{A}$ & 0 & $\mathrm{~N} / \mathrm{A}$ & 0 & $\mathrm{~N} / \mathrm{A}$ & \\
\hline "Otro motivo" & \multicolumn{2}{|l|}{$\begin{array}{l}84(35.59 \%) \\
(29.5-41.7 \%)\end{array}$} & $\begin{array}{c}2.17 \\
(0- \\
6.4 \%)\end{array}$ & 7 & $\begin{array}{c}8,3 \\
(2.4- \\
14.2 \%)\end{array}$ & $\begin{array}{r}47,6 \\
5\end{array}$ & $\begin{array}{c}47,61(36,9 \\
58,3 \%)\end{array}$ & \\
\hline & $\begin{array}{l}\text { BIRADS } \\
3\end{array}$ & $95 \%)$ & $\begin{array}{c}\text { BIRADS } \\
4\end{array}$ & $\begin{array}{l}\%(I C \\
95 \%)\end{array}$ & $\begin{array}{c}\text { BIRADS } \\
5\end{array}$ & $\%$ (IC 95\%) & $\begin{array}{l}\text { BIRADS } \\
6\end{array}$ & $\begin{array}{l}\text { \% (IC } \\
95 \%)\end{array}$ \\
\hline $\begin{array}{l}\text { Tamizaje de } \\
\text { pacientes con alto } \\
\text { riesgo }\end{array}$ & $\begin{array}{r}28,2 \\
42\end{array}$ & $\begin{array}{l}(14.7 \\
3 \%\end{array}$ & 3 & $\begin{array}{r}7.69(0- \\
16.1 \%)\end{array}$ & 1 & $\begin{array}{r}2,56(0- \\
7,5 \%)\end{array}$ & 1 & $\begin{array}{c}2,56 \\
(0- \\
7,5 \%)\end{array}$ \\
\hline $\begin{array}{l}\text { Estadiaje de } \\
\text { reciente } \\
\text { diagnóstico }\end{array}$ & 0 & /A & 1 & $\begin{array}{r}2.27(0- \\
6,7 \%)\end{array}$ & 6 & $\begin{array}{c}13.63 \% \\
(3,5-23,8 \%)\end{array}$ & 37 & $\begin{array}{l}84(73,3 \\
-94,9 \%)\end{array}$ \\
\hline $\begin{array}{l}\text { Evaluación de la } \\
\text { terapia } \\
\text { neoadyuvante }\end{array}$ & 0 & $\overline{A A}$ & 0 & $\mathrm{~N} / \mathrm{A}$ & 0 & $\mathrm{~N} / \mathrm{A}$ & 9 & $(-)$ \\
\hline $\begin{array}{l}\text { Carcinoma } \\
\text { primariooculto }\end{array}$ & 0 & $/ \mathrm{A}$ & 0 & $\mathrm{~N} / \mathrm{A}$ & 0 & $\mathrm{~N} / \mathrm{A}$ & 0 & $\mathrm{~N} / \mathrm{A}$ \\
\hline $\begin{array}{l}\text { Sospecha de } \\
\text { recurrencia local } \\
\text { cuando la biopsia } \\
\text { no pueda ser } \\
\text { realizada }\end{array}$ & 0 & $\overline{A A}$ & 0 & $\mathrm{~N} / \mathrm{A}$ & 1 & $(-)$ & 0 & $\mathrm{~N} / \mathrm{A}$ \\
\hline $\begin{array}{l}\text { Resultados } \\
\text { incongruentes } \\
\text { entre la } \\
\text { mamografía y el } \\
\text { ultrasonido }\end{array}$ & $\begin{array}{r}15 . \\
3\end{array}$ & $8(0$ & 3 & $\begin{array}{l}30,56 \\
(5.7- \\
55.9 \%)\end{array}$ & 1 & $\begin{array}{l}7,69(0 \\
22,2 \%)\end{array}$ & 0 & $\mathrm{~N} / \mathrm{A}$ \\
\hline $\begin{array}{l}\text { Evaluación de } \\
\text { extensión }\end{array}$ & 0 & $/ A$ & 1 & $\begin{array}{r}2,17(0- \\
6,4 \%)\end{array}$ & 5 & $\begin{array}{c}10,86(1.9 \\
19.9 \%\end{array}$ & 37 & $\begin{array}{c}80,43(69 \\
91,9 \%)\end{array}$ \\
\hline $\begin{array}{l}\text { Invasión a la fascia } \\
\text { profunda. }\end{array}$ & 0 & $\bar{A}$ & 0 & $\mathrm{~N} / \mathrm{A}$ & 0 & $\mathrm{~N} / \mathrm{A}$ & 0 & $\mathrm{~N} / \mathrm{A}$ \\
\hline $\begin{array}{l}\text { Bordes positivos } \\
\text { posterior a la } \\
\text { cuadrantectomía. }\end{array}$ & 0 & $\overline{A A}$ & 0 & $\mathrm{~N} / \mathrm{A}$ & 0 & $\mathrm{~N} / \mathrm{A}$ & 0 & $\mathrm{~N} / \mathrm{A}$ \\
\hline "Otro motivo" & $\begin{array}{r}10,7 \\
17\end{array}$ & $\begin{array}{l}1(4,1 \\
3 \%)\end{array}$ & 9 & $\begin{array}{c}10,71(4,1 \\
17,3 \%)\end{array}$ & 10 & $\begin{array}{r}11,90(5- \\
18.8 \%)\end{array}$ & 8 & $\begin{array}{r}9,52(3.2 \\
15.8 \%)\end{array}$ \\
\hline
\end{tabular}

Fuente:Datos propios de los autores. 
Tabla 4. Valor predictivo positivo para cáncer de mama en resonancias para BIRADS 4 y 5 reportados por resonancia magnética.

\begin{tabular}{llll} 
& Resultado BIRADS & Cáncer & VPP \\
\hline BIRADS 4 & 17 & 5 & $29 \%(7.8-51.1 \%)$ \\
\hline BIRADS 5 & 24 & 19 & $79 \%(62.9-95.4 \%)$ \\
\hline BIRADS 4 y 5 & 41 & 25 & $60 \%(46-75.9 \%)$ \\
\hline
\end{tabular}

Los datos presentados entre paréntesis () representan el intervalo de confianza $>95 \%$.

Fuente: Datos propios de los autores.

Tabla 5. Cambio de conducta y valores predictivos positivos para las categorías BI-RADS 4,5,6 y valor predictivo positivo de cambio de conducta.

\begin{tabular}{|c|c|c|c|c|c|}
\hline Indicación & (n) & $\begin{array}{l}\text { VPP BI - } \\
\text { RADS } 4\end{array}$ & $\begin{array}{l}\text { VPP BI - } \\
\text { RADS } 5\end{array}$ & $\begin{array}{l}\text { VPP BI - } \\
\text { RADS } 6\end{array}$ & $\begin{array}{l}\text { VPP de } \\
\text { cambio de } \\
\text { conducta }\end{array}$ \\
\hline $\begin{array}{l}\text { Tamizaje de pacientes } \\
\text { con alto riesgo }\end{array}$ & 5 & $\begin{array}{l}7.69 \%(0- \\
16.1 \%)\end{array}$ & $\begin{array}{l}2.56 \%(0- \\
7.5 \%)\end{array}$ & $\begin{array}{l}2.56 \% \quad(0- \\
7.5 \%)\end{array}$ & $\begin{array}{l}12.82 \%(2.3- \\
23.3 \%)\end{array}$ \\
\hline $\begin{array}{lll}\text { Resultados } & & \\
\text { incongruentes } & \text { entre } & \text { la } \\
\text { mamografía } & \text { y } & \text { el } \\
\text { ultrasonido } & & \end{array}$ & 4 & $\begin{array}{l}23.07 \%(0,- \\
46 \%)\end{array}$ & $\begin{array}{ll}07.69 & (6.8- \\
22.2 \%) & \end{array}$ & $\mathrm{N} / \mathrm{A}$ & $\begin{array}{l}30.76 \%(5.7- \\
55.9 \%)\end{array}$ \\
\hline "otro motivo" & 27 & $\begin{array}{l}10.71 \% \quad(0- \\
041-17.3 \%)\end{array}$ & $\begin{array}{l}12.04 \% \\
0.18 .8 \%)\end{array}$ & $9.63 \%$ & $\begin{array}{l}32.53 \% \\
(22.2- \\
42.1 \%)\end{array}$ \\
\hline
\end{tabular}

-(n)=número de pacientes en quienes se cambia la conducta según categorías BI-RADS 4,5,6.

-()$=$ intervalo de confianza $=95 \%$

-Se define cambio en la conducta como: realización de biopsia u otra intervención.

Fuente: Datos propios de los autores. 
En el resto pudo cambiar la conducta médica, pero fue realizada en pacientes con diagnóstico confirmado como neoplasia maligna mediante otras alternativas diagnósticas e histopatológicas, en estos casos, su uso fue justificado para evaluar la extensión o la respuesta al tratamiento, sin embargo, actualmente no hay soporte de la literatura sobre estas indicaciones y la única que tiene soporte, es el cribado de alto riesgo en donde la conducta tan solo cambió en un $12.82 \%$ en la población de estudio.

Otro hallazgo importante, fue que a muchas pacientes asignadas con BI-RADS 4 y 5 en quienes se les solicito el estudio de resonancia magnética bajo los motivos de "estadiaje de reciente diagnóstico" y "evaluación de la extensión" se les realizo el estudio antes de tener confirmación histológica.

Esto puede ser probablemente explicado por el temor en la práctica a demoras con el diagnóstico debido a los diferentes problemas administrativos y otras fallas en el sistema de salud, que puede motivar a práctica no soportada en evidencia y que eventualmente puede producir un aumento en la demanda de dicho estudio similar a lo que ocurre en otros países ${ }^{18}$ por tanto, se deben reunir los elementos necesarios antes de solicitar el estudio y se debe tener una mayor adherencia a las indicaciones que muestran mayor evidencia.

Respecto a resultados de estudios que reportan hallazgos sobre el valor predictivo de la resonancia magnética de mama en algunas indicaciones, un estudio realizado en año 2011 por Yau et al. en 204 pacientes para determinar la utilidad de la resonancia magnética como herramienta para resolver los dilemas cuando los resultados entre la mamografía y el ultrasonido son incongruentes, se obtuvieron 42 pacientes catalogados como BI-RADS 4 y 5 (VPP de $20,58 \%$ ) siendo menor que en el estudio que se presenta en este artículo, hallazgo que puede explicarse por las diferencias en prevalencias de patología mamaria debidas a las características de los centros de atención, siendo la unidad de mama un centro de referencia con mayor prevalencia de atención de patología mamaria.

Por otra parte, la prevalencia de patología maligna fue en este grupo de $33 \%$, dado por 14 de los 42 pacientes BI-RADS 4 y 5 que resultaron positivos para hallazgos malignos por patología, el valor predictivo positivo fue de $33 \%$, valor similar al de este estudio el cual fue de $30.76 \% .^{23}$ Otro estudio similar realizado por Moy et al. en el 2009, revisó 115 resonancias en pacientes con "hallazgos incongruentes entre ma- mografía y ecografía en mamografía" y encontraron que de 115 pacientes 15 fueron clasificadas como BIRADS 4 o 5 (VPP 13,04\%) y de estas, 6 dieron positivo para malignidad por histopatología encontrando un VPP de 40\% (IC95=19.1\%-66.8\%).

En su estudio la prevalencia de cáncer calculada fue de $5.51 \%$ y concluyeron que podría ser una buena herramienta para la resolución de dilemas diagnósticos cuando hay hallazgos que no concuerdan en la mamografía y ecografía, sin embargo, se debe tener en cuenta que es una modalidad costosa, con alta tasa de falsos positivos y una alta tasa de frecuencia de hallazgos incidentales. ${ }^{24}$

En un metaanálisis que incluyó 2316 casos, realizado para determinar la exactitud diagnóstica de la resonancia para resolver dilemas cuando los hallazgos son no concuerdan entre la ecografía y la mamografía realizado por Barbaraet al publicado en el 2016, obtiene un valor predictivo positivo variable para lesiones malignas que oscila entre el $25 \%$ al $96.4 \%$ y un valor predictivo positivo promedio de $56 \%$ y concluyen que a pesar de que la resonancia tiene un excelente rendimiento cuando los hallazgos son no concordantes entre las imágenes convencionales, se debe considerar la heterogeneidad de la prevalencia de la enfermedad, lo cual impacta directamente en el VPP y adicionalmente indican que los criterios para resolver dilemas diagnósticos cuando los resultados son no concordantes entre la mamografía y el ultrasonido deben ser mejor definidos. ${ }^{25}$

En un estudio realizado en el 2018 por Vreemann et al. donde se evalúa el rendimiento diagnóstico de la resonancia magnética para evaluar pacientes de alto riesgo de cáncer donde incluyen 8818 resonancias para tamizaje y 6245 mamografías en 2463 mujeres, encuentran un valor predictivo positivo para realizar biopsia de 22\% (IC95\%=15-26\%) en la primera resonancia realizada a las pacientes con alto riesgo y consecuentemente un valor predictivo positivo que alcanza un valor de $34 \%$ (IC95=28-40\%) en seguimientos posteriores. ${ }^{26}$

En el estudio que se presenta en este artículo se encuentran valores predictivos de cambios en la conducta (aquellas pacientes a quienes se les realizó biopsia por hallazgos BI-RADS 4 o 5) de $12.82 \%$ (IC95\%=2.3-23.3\%), esta cifra es considerablemente baja respecto a lo reportado en la literatura y en nuestro marco de estudio y puede ser explicado por la forma heterogénea en la que se define como "alto riesgo en las pacientes". 
Si bien "alto riesgo" se define como el riesgo de más del $25 \%$ de tener cáncer de mama o ser portadoras de mutaciones genes BRCA1/2, TP53 entre otras mutaciones. $^{27}$

El cribado genético en Colombia para cáncer de mama no es una práctica extendida y durante muchos años se privilegió el cribado para mutaciones BRCA 1 y 2 lo cual no excluye la presencia de mutaciones en otros genes que también pueden estar relacionados con el cáncer de mama hereditario; Especialmente en Colombia y en la mayor parte de países de Latinoamérica, donde el perfil mutacional es diferente y altamente heterogéneo. ${ }^{28,29}$

Este estudio es uno de los primeros en su naturaleza en el país, permitió reconocer el valor predictivo de la resonancia magnética de mama bajo indicaciones diferentes a alto riesgo ${ }^{30}$; el alto costo de esta modalidad y su pobre rendimiento predictivo, demostrado en este trabajo, a pesar de una elevada prevalencia de patología maligna y de patología mamaria en general, puede sugerir limitar el uso de esta modalidad en otras indicaciones que no sean pacientes con riesgo de cáncer de mama mayor del $25 \%$ en toda la vida, como se ha concluido en la literatura. ${ }^{31}$

Adicionalmente, el bajo VPP aun cuando la indicación es alto riesgo, hace necesario recomendar aclarar las razones para su solicitud y una exploración adecuada de antecedentes y factores genéticos, con el fin de mejorar la probabilidad pretest y así mejorar el rendimiento predictivo de la misma para lesiones malignas.

Este estudio tiene limitaciones secundarias al diseño retrospectivo del mismo y propias de las fuentes de información, en las que es posible que muchas indicaciones no fueran adecuadas en su descripción en las historias clínicas.

Adicionalmente, se calculó exclusivamente el valor predictivo positivo, y no otras características de su rendimiento diagnóstico, dada la ausencia de reporte histopatológico en pacientes con lesiones BIRADS 0 a 3.

\section{Conclusiones}

La RM de mama tiene un valor predictivo positivo bajo para las indicaciones descritas en la literatura en un centro de referencia para el diagnóstico, estudio y manejo de patología mamaria en Colombia.

Razones como barreras administrativas, temor en la práctica clínica y otras barreras de acceso deben ser estudiadas en el futuro para determinar si pueden explicar su sobre uso y los hallazgos de este estudio.

Son necesarios más estudios que permitan determinar no solo el valor predictivo positivo de estas indicaciones, sino, su exactitud diagnóstica.

Identificación de la responsabilidad del autor y grado de contribución por su trabajo:

MG-CD - Idea principal, revisión y supervisión del estudio.

IJ, SV- Recolección de datos, análisis e interpretación de datos, redacción del borrador y del artículo final.

KE - Diseño del estudio, interpretación de datos, análisis, redacción, borrador y borrador final.

Grado de contribución de los autores: Los autores declaran haber contribuido de forma similar en la idea, diseño del estudio, análisis y redacción del artículo final. Conflictos de interés: Los autores no declaran conflictos de interés en esta investigación.

Fuente de financiamiento: Recursos propios. 


\section{Bibliografía}

1. Ministerio de Salud y Protección Social, Instituto Nacional de Cancerología. El cáncer de mama: un problema creciente en Colombia. Hechos y Acciones [Internet]. 2012;4(2):1-2. Available from: https:// www.cancer.gov.co/files/libros/archivos/95685f345e64aa9fofece8a589b5acc3_BOLETIN HECHOS Y ACCIONES MAMA.PDF

2. GLOBOCAN 2018 Graph production: IARC.

3. Globocan Observatory W, IARC, (IARC) IA for R on C, (WHO) WHO. Breast Cancer. Source: Globocan 2018. Int Agency Res Cancer. 2019;876:2018-9.

4. Institude N cancer. Advances in Breast Cancer Research [Internet]. 2020. Available from: https://www.cancer.gov/types/breast/ research

5. Zapolski. The ACR BI-RADS $®$ Experience: Learning From History. J Am Coll Radiol. 2008;23(1):1-7.

6. Murphy CD, Lee JM, Drohan B, Euhus DM, Kopans DB, Gadd MA, et al. The American Cancer Society guidelines for breast screening with magnetic resonance imaging: An argument for genetic testing. Cancer. 2008;113(11):3116-20.

7. Kuhl CK, Mielcareck P, Klaschik S, Leutner C, Wardelmann E, Gieseke J, et al. Dynamic breast MR imaging: Are signal intensity time course data useful for differential diagnosis of enhancing lesions? Radiology. 1999;211(1):101-10.

8. Rausch DR, Hendrick RE. How to optimize clinical breast MR imaging practices and techniques on your 1.5-T system.

Radiographics. 2006;26(5):1469-84.

9. Surov A, Meyer HJ, Wienke A. Can apparent diffusion coefficient (ADC) distinguish breast cancer from benign breast findings? A meta-analysis based on 13847 lesions. BMC Cancer. 2019;19(1):114.

10. Vanvuren C. New choice health. Cost of Medical Procedures in the U.S. , MRI.

11. Jung J, Kang H, Kim M, Lee W, Min K, Han M, et al. Reaction to Gadolinium-based. RSNA Radiol. 2012;264(2).

12. Gulani V, Calamante F, Shellock FG, Kanal E, Reeder SB. Gadolinium deposition in the brain: summary of evidence and recommendations. Lancet Neurol [Internet]. 2017;16(7):564-70. Available from: http://dx.doi.org/10.1016/S1474-4422(17)30158-8

13. Hellman RN. Gadolinium-induced nephrogenic systemic fibrosis. Semin Nephrol [Internet]. 2011;31(3):310-6. Available from: http:// dx.doi.org/10.1016/j.semnephrol.2011.05.010

14. Menezes GLG, Knuttel FM, Stehouwer BL, Pijnappel RM, Van Den Bosch MAAJ. Magnetic resonance imaging in breast cancer: A literature review and future perspectives. World $\mathrm{J}$ Clin Oncol. 2014;5(2):61-70.

15. Feig S. Comparison of Costs and Benefits of Breast Cancer Screening with Mammography, Ultrasonography, and MRI. Obstet Gynecol Clin North Am [Internet]. 2011;38(1):179-96. Available from: http://dx.doi.org/10.1016/j.ogc.2011.02.009

16. Ross J, Thompson JS, Bailey A. Nuclear Magnetic Resonance Imaging and Evaluation of Human Breast Tissue : Preliminary Clinical Trials. Radiology. 1982;143:195-205.

17. Miller JW, Sabatino SA, Thompson TD, Breen N, White MC, Ryerson $A B$, et al. Breast MRI use uncommon among U.S. women. Cancer Epidemiol Biomarkers Prev. 2013;22(1):159-66.
18. Stout NK, Nekhlyudov L, Li L, Malin ES, Ross-Degnan D, Buist DSM, et al. Rapid increase in breast magnetic resonance imaging use trends from 2000 to 2011. JAMA Intern Med. 2014;174(1):11421.

19. Wernli KJ, DeMartini WB, Ichikawa L, Lehman CD, Onega T, Kerlikowske $\mathrm{K}$, et al. Patterns of breast magnetic resonance imaging use in community practice. JAMA Intern Med. 2014;174(1):125-32.

20. González EM, Dávila VC, Juan SI, Veieira SS EO. Federación Ecuatoriana de Radiología e Imagen Revista Federación Ecuatoriana de Radiología e Imagen. 2019;12(1):volumen 12, No.1.

21. Torres D, Rashid MU, Gil F, Umana A, Ramelli G, Robledo JF, et al. Guía de práctica clínica (GPC) para la detección temprana, tratamiento integral, seguimiento y rehabilitación del cáncer de mama. Vol. 12 Suppl 1, Breast Cancer Research and Treatment. 2013. S54-69 p.

22. Mahoney MC, Gatsonis C, Hanna L, DeMartini WB, Lehman C. Positive predictive value of BI-RADS MR imaging. Radiology. 2012;264(1):51-8.

23. Yau EJ, Gutierrez RL, Demartini WB, Eby PR, Peacock S, Lehman $\mathrm{CD}$. The utility of breast MRI as a problem-solving tool. Breast J. 2011;17(3):273-80.

24. Moy L, Elias K, Patel V, Lee J, Babb JS, Toth HK, et al. Is breast $\mathrm{MRI}$ helpful in the evaluation of inconclusive mammographic findings? Am J Roentgenol. 2009;193(4):986-93.

25. Bennani-Baiti B, Bennani-Baiti N, Baltzer PA. Diagnostic performance of breast magnetic resonance imaging in non-calcified equivocal breast findings: Results from a systematic review and meta-analysis. PLoS One. 2016;11(8):99-100.

26. Vreemann S, Gubern-Mérida A, Schlooz-Vries MS, Bult P, Van Gils $\mathrm{CH}$, Hoogerbrugge N, et al. Influence of risk category and screening round on the performance of an MR imaging and mammography screening program in carriers of the BRCA mutation and other women at increased risk. Radiology. 2018;286(2):443-51.

27. Instituto Nacional de Cancerología ESE. Guía de práctica clínica (GPC) para la detección temprana, tratamiento integral, seguimiento y rehabilitación del cáncer de mama. Bogotá D.C. 2013. $1-930 \mathrm{p}$.

28. Camacho CMCRG. Estudio técnico de la prueba "Perfil Colombia " para tamización de mutaciones en pacientes con cáncer de mama en el marco del procedimiento técnico científico y participativo de exclusiones Reporte No . 1150 Septiembre de 2019. IETS. 2019;(1150).

29. Cortés C, Rivera AL, Trochez D, Solarte M, Gómez D, Cifuentes $L$, et al. Mutational analysis of BRCA1 and BRCA2 genes in women with familial breast cancer from different regions of Colombia. Hered Cancer Clin Pract. 2019;17(1):1-10.

30. Gunawardena D. Current Status of Breast MRI -Clinical applications. Sri Lanka J Radiol. 2015;1(0):1.

31. Ahern CH, Shih YCT, Dong W, Parmigiani G, Shen Y. Cost-effectiveness of alternative strategies for integrating MRI into breast cancer screening for women at high risk. $\mathrm{Br} \mathrm{J}$ Cancer [Internet]. 2014;111(8):1542-51. Available from: http://dx.doi.org/10.1038/ bjc. 2014.458 